\title{
Cosmetic camouflage of visible skin lesions enhances life quality indices in leprosy as in vitiligo patients: an effective stigma reduction strategy
}

\author{
P. Narasimha $\mathrm{Rao}^{\mathrm{a}}$, Manogna Vellala ${ }^{\mathrm{b}}$, Arun Raghav Potharaju ${ }^{\mathrm{c}} \&$ \\ K. Udaya Kiran ${ }^{\mathrm{d}}$ \\ ${ }^{\text {a } P r o f e s s o r ~ o f ~ D e r m a t o l o g y, ~ B h a s k a r ~ M e d i c a l ~ C o l l e g e, ~ T e l a n g a n a, ~ I n d i a ~}$ \\ ${ }^{\mathrm{b}}$ Institute of Preventive Medicine, Hyderabad, India \\ ${ }^{\mathrm{c}}$ Deccan College of Medical Sciences, Hyderabad, India \\ ${ }^{\mathrm{d}}$ FCGP, Director, Medilab, Hyderabad, India
}

Submitted 1 September 2020; Accepted 19 October 2020

\begin{abstract}
Summary The use of cosmetic camouflage for blemishes on skin of affected people is known to improve their Dermatology Life Quality Index (DLQI). Although the use and benefits of cosmetic camouflage is known and reported in vitiligo, its value in leprosy, where visible skin lesions are a reason for stigma and discrimination, is yet to be explored. A key resolution of the WHO Global Leprosy Strategy 2016-2020 is to stop discrimination and promote inclusion of persons affected by leprosy into society. Improving the DLQI of leprosy patients with the use of cosmetic camouflage is an effort in that direction.

Objectives: We undertook this study to examine the change brought about by cosmetic camouflage in the DLQI of leprosy patients with visible skin lesions and, to compare the results with its use in vitiligo, as both are disorders associated with pigment dilution in the skin.

Methods: The DLQI score was calculated for 9 consenting leprosy and 14 vitiligo patients at the time of enrolment. All patients were taught the simple technique of cosmetic camouflage of their skin patches on exposed parts of the body, such as face and arms/forearms. The DLQI scoring was calculated again after one month of use of cosmetic camouflage and compared with the baseline value.

Results: The mean DLQI improved significantly $(p<0.0001)$ after the use of cosmetic camouflage in both vitiligo and leprosy groups, indicating the efficacy of camouflage in improving the quality of life. In addition, it was observed that the mean improvement in DLQI was higher in the leprosy group $(14.67 \pm 3.87)$ than in the vitiligo group $(8.64 \pm 2.96)$, the difference being statistically significant $(p<0.05)$, suggesting that camouflage has a very high impact on the quality of life in leprosy.

Conclusions: Leprosy-related stigma and discrimination are pervasive in almost all cultures of the world. Such stigma affects many aspects of social participation. The WHO global strategy document 2016-2020 advocates inclusion of at least one stigma reduction strategy in all leprosy programme plans. Use of cosmetic camouflage for
\end{abstract}

Correspondence to: Dr. P. Narasimha Rao, B48, Income tax colony, Mehdipatnam, Hyderabad 500028, India (e-mail: dermarao@hotmail.com; dermarao@gmail.com) 
visible skin lesions on exposed parts of the body in leprosy patients could be one such strategy.

Keywords: Cosmetic camouflage, DLQI, Leprosy, stigma

\section{Introduction}

Skin diseases have been recognized as having a detrimental effect on the quality of life (QoL) and social relationships, self-image and self-esteem in ways that are different from other nondermatological diseases. ${ }^{1}$ Leprosy adversely affects the QoL of those affected, more severely in persons with visible deformities. ${ }^{2,3}$ The specific effect of visible skin lesions of leprosy on stigma and QoL in a patient have not been studied. The stigma associated with leprosy from ancient times was so severe that the afflicted were segregated into separate colonies. ${ }^{4,5}$ Vitiligo patients in India also have a long history of social discrimination and stigma that is known to severely affect their lives. ${ }^{6}$

Many studies document the effects of stigma in leprosy, but few discuss or evaluate specific solutions. ${ }^{4,6,7}$ It has been suggested that social stigmatization is greater when disfigurements are located on visible parts of the body and any strategy to make these less visible or invisible during times of social interaction helps to prevent such stigmatization and the resulting distress. ${ }^{8}$ In leprosy, discoloured skin lesions on exposed parts could be the cause for social stigma. Cosmetic camouflage is a simple, safe and inexpensive method to make these lesions less visible during social contact.

The Dermatology Life Quality Index (DLQI) assessment score was formulated as a questionnaire to grade the impact of various skin disorders on the quality of life (QoL). ${ }^{9-12}$ Studies have been carried out on the impact of vitiligo on the DLQI of patients and it was found to be significantly lower, ${ }^{13,14}$ and further studies noted improvement in DLQI with the use of cosmetic camouflage. ${ }^{15,16}$ In leprosy, however, although there are papers on overall impact of leprosy and its disabilities on DLQI, ${ }^{2,3}$ there are no published studies specific to the impact of visible skin lesions on DLQI or its improvement by cosmetic camouflage. The present study attempts to evaluate the effect of cosmetic camouflage on DLQI in leprosy and vitiligo patients with visible skin lesions and make comparisons between the two groups.

\section{Methods}

This study was carried out at the Department of Dermatology, Bhaskar Medical College, Yenkapally, RR district, Telangana, India. Institutional ethical clearance was obtained prior to starting the study. A total of 9 patients with leprosy and 14 patients with vitiligo, with lesions on visible areas of the upper limbs and face were included in the study over a period of 6 months, from September 2014 to March 2015, after written informed consent, including permission for photography was obtained from all patients. At entry, a detailed clinical examination was carried out and findings recorded. Patients with leprosy were also assessed for the presence of lepra reactions.

In order to administer the DLQI questionnaire to each participant for the present study, copyright permission was obtained from Cardiff University, United Kingdom. The questionnaire consists of ten questions with graded answers according to the Likert scale and is divided into five sections pertaining to symptoms and feelings; daily activity; leisure; work and school; and personal relationships (Figure 1). High scores indicate poor quality of life while low scores 


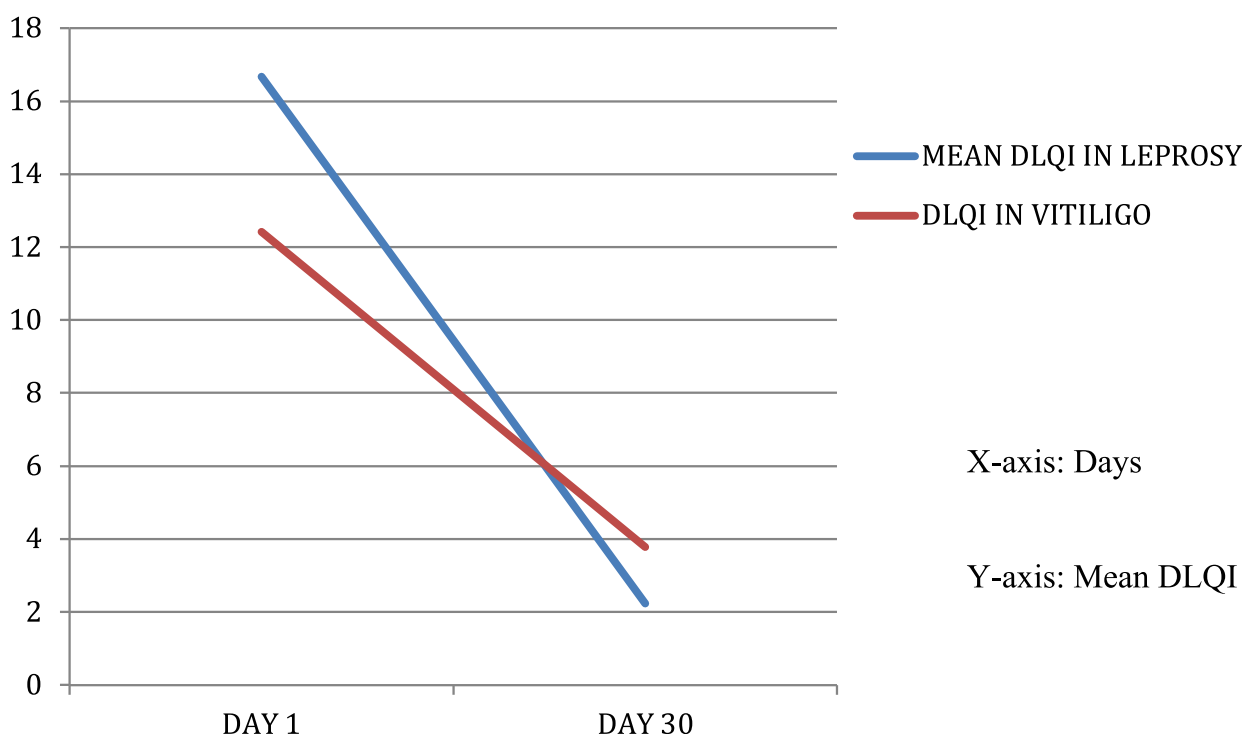

Figure 1 A line diagram showing the changes in DLQI before and after the use of camouflage. The blue line represents the change in mean DLQI in patients in the leprosy group. The red line represents change in patients in the vitiligo group.

indicate good quality. The questionnaire was modified for the present study by the omission of the question related to ease of topical treatment as it was not relevant to the leprosy patient group. The DLQI questionnaire was administered to all participants at the time of entry and scores recorded.

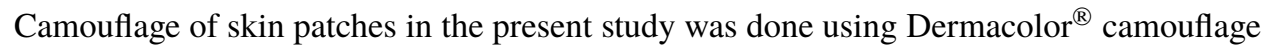
creme kit (Kryolan, USA), to obtain a temporary and removable near skin colour match with surrounding skin. ${ }^{17}$ Dermacolor is available in a wide range of shades suitable for all skin types and are intermixable to give the make-up a natural appearance when applied correctly. It is especially suitable for face and neck, and can be fixed using a powder to make it stay for many hours, with easy reapplication as and when needed. It takes about 6 minutes to cover a palm sized patch. Patients were educated in the clinic on its use and shown how to apply camouflage on the skin lesions themselves. All patients were provided with the camouflage kit in shades appropriate to their skin colour, along with the fixing powder, and were advised to apply the product daily for 30 days during periods of social contact and to cover all patches on exposed areas of the body. At the end of 30 days, the DQLI was reassessed and results compared with the DLQI scores recorded at the beginning of the study. In addition to camouflage, appropriate treatment was given to all patients for their disease, without interfering with the camouflage. Clinical photographs were taken before and after application of camouflage.

\section{Results}

Leprosy study group: A total of 9 patients with leprosy (male 5; female 4), with skin patches on the face and forearm, were included in the study. The ages of the patients ranged from 16 to 47 years, with a mean age of 28.9 years. The most common diagnosis was Borderline Tuberculoid (BT) leprosy in $6(66.6 \%)$ patients, followed by Tuberculoid (TT) in 2 and Indeterminate leprosy in 1 patient. Skin lesions of leprosy were seen on the face in 7 patients and on the 

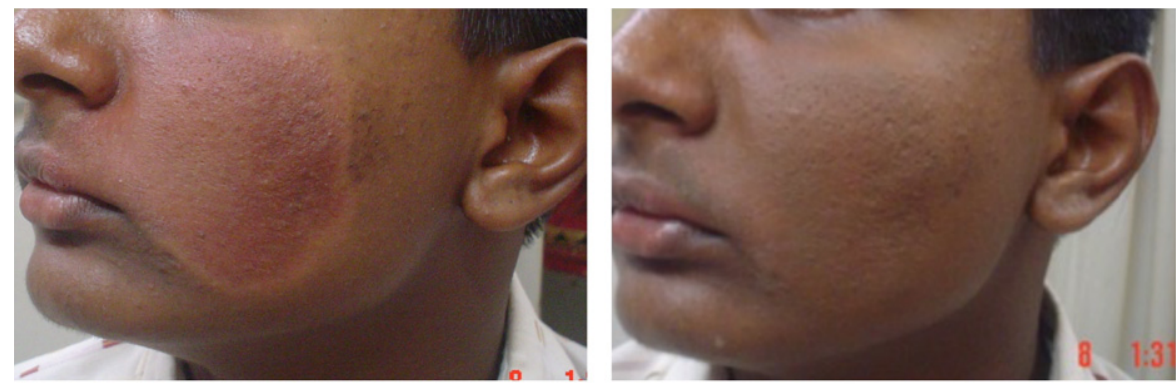

Figure 2 BT patch on cheek before and after effective cosmetic camouflage.
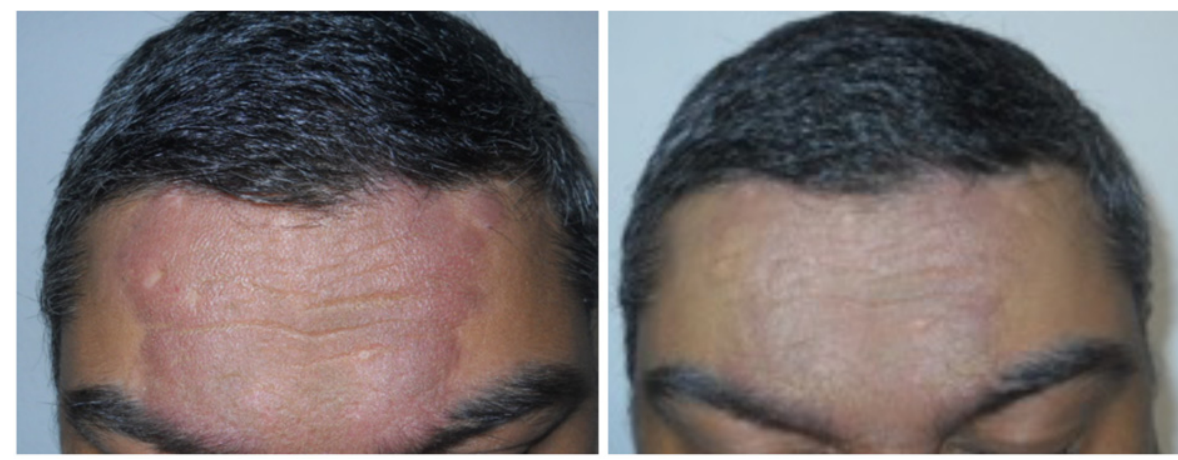

Figure 3 BT patch on forehead in type 1 reaction before and after cosmetic camouflage.

forearm in 2 patients. Two patients with BT leprosy had associated Type 1 reaction involving the skin patches on the face. Cosmetic camouflage was accepted well by all the patients, including those who had type 1 reaction in the facial patches, with camouflage making the patches merge with the skin and mask the erythema (Figures 2, 3 and 5).

DLQI assessment: The DLQI score for various sections is shown in Table 1. Overall, the total mean DLQI of leprosy patients before the use of camouflage was $16.67 \pm 3.87$ and reduced to $2.23 \pm 1.16$ after its use, the difference $(14.67 \pm 3.87)$ was statistically highly significant $(p<0.0001)$. When the different parameters of quality of life were assessed, the difference between the parameters symptoms and feelings, daily activity, leisure before and after one month use of camouflage was highly significant at a $p$ value $<0.0001$ and the difference for parameters work and school and personal relationships were statistically significant at a $p$ value $<0.05$.

Vitiligo study group: A total of 14 patients (male 10; female 4) with vitiligo, with lesions on exposed areas of the body, were included in the study. The ages ranged from 15 to 50 years, with a mean age of 26.07 years. Eight patients in this group had facial patches and the remaining 6 had patches on exposed areas of the upper limbs.

DLQI assessment: The mean DLQI score for various sections is shown in Table 2. The overall mean DLQI of the patients before using camouflage was $12.42 \pm 4.48$ and after using camouflage was $3.78 \pm 1.52$. The mean difference in DLQI of $8.64 \pm 2.96$, was highly significant $(p<0.0001)$ (Figure 4). When different parameters of DLQI were assessed, the mean DLQI score before the use of camouflage was very high for all parameters, symptoms and 
Table 1. DLQI before and after camouflage in leprosy patients

\begin{tabular}{cccc}
\hline Section of DLQI & $\begin{array}{c}\text { DLQI score before Camouflage } \\
(\text { Mean } \pm \text { SD) }\end{array}$ & $\begin{array}{c}\text { DLQI after 30 days of use of } \\
\text { Camouflage (Mean } \pm \text { SD) }\end{array}$ & $P$ value \\
\hline Symptoms and feelings & $4.56 \pm 1.13$ & $0.78 \pm 0.67$ & $<0.0001$ \\
Daily activities & $4.22 \pm 1.20$ & $0.33 \pm 0.24$ & $<0.0001$ \\
Leisure & $4.11 \pm 1.62$ & $0.56 \pm 0.53$ & $<0.0001$ \\
Work and school & $1.22 \pm 0.43$ & $0.22 \pm 0.15$ & $<0.05$ \\
Personal relationships & $2.56 \pm 1.67$ & $0.33 \pm 0.24$ & $<0.05$ \\
Total & $16.67 \pm 3.87$ & $2.23 \pm 1.16$ & $<0.0001$ \\
\hline
\end{tabular}
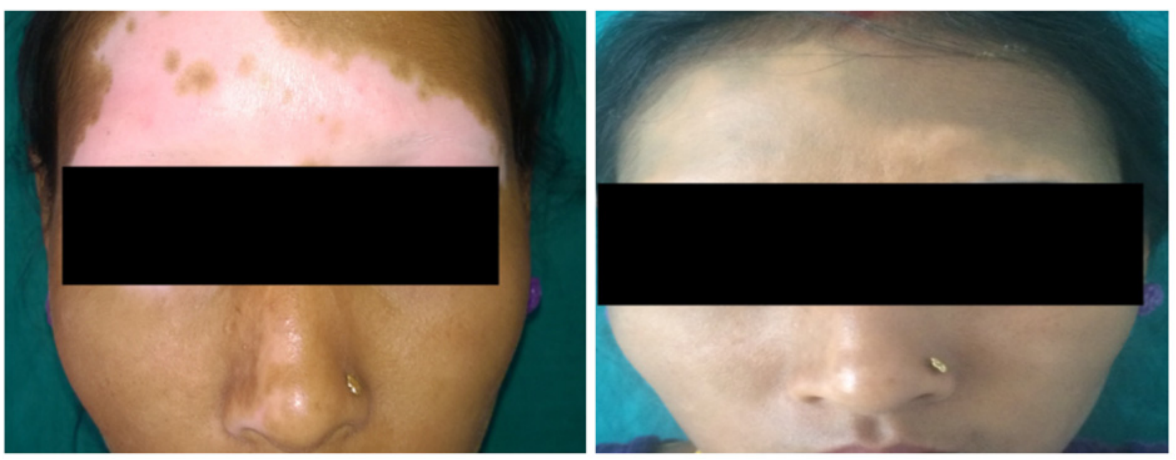

Figure 4 Vitiligo patch on forehead before and after cosmetic camouflage.

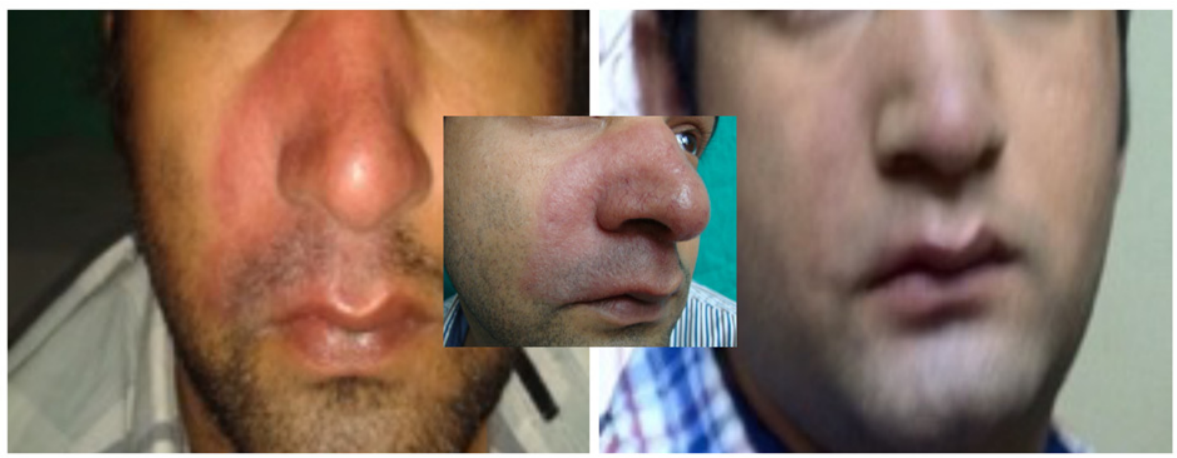

Figure 5 Cosmetic camouflage of residual persistent erythema on face and upper lip in a MDT completed patient.

feelings, daily activity, leisure, work and school, and personal relationships when compared to values after its use for a month, and the difference was statistically significant $(p<0.05)$ for all parameters.

\section{Discussion}

Appearance is one of the most powerful factors influencing social interactions with others and patients with disfigurements experience very real stigma from society on a daily basis. ${ }^{18}$ Cosmetic camouflage when properly applied can help improve the patient's appearance 
Table 2. DLQI before and after camouflage in vitiligo patients

\begin{tabular}{cccc}
\hline Section of DLQI & $\begin{array}{c}\text { DLQI score before Camouflage } \\
(\text { Mean } \pm \text { SD) }\end{array}$ & $\begin{array}{c}\text { DLQI after 30 days of use of } \\
\text { Camouflage (Mean } \pm \text { SD) }\end{array}$ & $P$ value \\
\hline Symptoms and Feelings & $0.93 \pm 0.27$ & $3.14 \pm 1.03$ & $<0.0001$ \\
Daily activity & $2.86 \pm 1.92$ & $0.86 \pm 0.95$ & $<0.0004$ \\
Leisure & $2.71 \pm 1.59$ & $0.79 \pm 0.80$ & $<0.0001$ \\
Work and School & $0.79 \pm 1.25$ & $0.00 \pm 0.00$ & $<0.0352$ \\
Personal relationships & $2.07 \pm 1.07$ & $0.64 \pm 0.74$ & $<0.001$ \\
Total & $12.42 \pm 4.48$ & $3.78 \pm 1.52$ & $<0.0001$ \\
\hline
\end{tabular}

considerably, thereby improving self-esteem and quality of life. ${ }^{19}$ Most camouflage cosmetics are inert, fragrance free and specially designed to be hypoallergenic, hence are gentle on the skin. ${ }^{20}$ Covering skin defects of contour and colour which are visible signs of the disease with camouflage minimizes stigmatization. ${ }^{21}$ Moreover, cosmetic camouflage improves the quality of life of patients without interfering with the treatment. ${ }^{22}$

\section{PERSISTENT FACIAL PATCHES IN LEPROSY}

Macular and hypopigmented leprosy lesions are known to occur anywhere on the body, but are often found on visible sites such as the face and lateral or dorsal aspects of extremities. ${ }^{23}$ In a cross sectional study from central India, of the 160 leprosy patients examined, 64 (40\%) presented with hypopigmented facial cutaneous lesions. ${ }^{24}$ Facial patches of leprosy can be both pale, flat, and macular type or red raised patches in reaction. ${ }^{25}$ Leprous involvement of the lips can present as a marked swollen and rigid appearance of the lips and hence cosmetically quite troublesome. ${ }^{26}$ In addition, late type 1 reactions (also known as reversal reaction) are known to occur up to 7 years after completion of MDT, ${ }^{27}$ which can keep the facial patches noticeable for months and years due to the associated erythema, in addition to hypopigmentation. When such lesions occur their visibility can lead to anticipated stigma in the patient, which refers to the fear of enacted stigma by society. Anticipated stigma is typically known to occur in patients with a dermatological condition that can be concealed. ${ }^{28}$ Stigma affects many aspects of the lives of leprosy patients, including mobility, interpersonal relationships, marriage, employment, leisure activities, and attendance at social and religious functions, ${ }^{29}$ and also of their family members. ${ }^{30,31}$ Many levels of interventions and strategies to fight stigma in skin diseases have been described of which changing the image of the disease is one of them. ${ }^{32}$ Leprosy is often portrayed as a disease with gross deformities and unsightly patches, so this image needs to change. This study found that cosmetic camouflage which masks the visible skin patches, is an effective intervention to improve both the image of the disease as well as the self-image of the patient and decrease social avoidance in patients with visible skin lesions.

\section{COSMETIC CAMOUFLAGE AS A TOOL AGAINST STIGMA}

Cosmetic camouflage was first documented as a modality of treatment to cover visible areas of disfigurement in patients suffering from burns following World war II. ${ }^{33}$ Its use was observed to improve the quality of life (QoL) in affected persons. The DLQI questionnaire is the most frequently used tool to evaluate QoL for patients with different dermatological conditions. Although the role of cosmetic camouflage in improving DLQI is reported in a variety of disfiguring and distressing dermatological conditions such as psoriasis, ${ }^{34}$ hand eczema, ${ }^{35}$ atopic dermatitis ${ }^{11}$ and vitiligo, ${ }^{12}$ its use in leprosy has not been reported till date, as confirmed 
in a thorough web search of the literature. It is known that leprosy patches can be present on exposed parts of the body and can persist for many years after the completion of MDT. While cosmetic camouflage was considered for other diseases with skin blemishes, the reason why it was never considered relevant for leprosy is unknown, and can only be contemplated upon, as it is one of the highly stigmatized diseases of mankind. ${ }^{36,37}$ As this was a pilot study in leprosy we decided to include patients with vitiligo which is a dermatological disease with known stigma $^{38-40}$ who are known to benefit from the use of camouflage, ${ }^{15,16}$ in order to assess the impact of cosmetic camouflage in leprosy, in direct comparison with vitiligo.

\section{KNOWN BENEFITS IN VITILIGO, PROVEN AGAIN IN LEPROSY}

Vitiligo and leprosy cause considerable impairment to various aspects of the life of the patients. Vitiligo is a condition wherein given the visibility and chronicity, stigmatization becomes a part of daily life in patients, which can impair QoL and lead to psycho-social stress. ${ }^{13,41}$ A study done in Assam, India observed that the mean DLQI was 4.40 in vitiligo patients with lesions on unexposed areas, while it was 10.25 in patients with lesions on exposed areas, indicating that DLQI differs with the sites of involvement. ${ }^{14}$ This is evidenced in the present study wherein the initial mean DLQI scores of the patients in both groups were high (vitiligo group $12.42 \pm 4.48$ and leprosy group $16.67+3.87$ ) as they had skin patches on exposed areas. Another reason for this difference could be the higher degree of stigma associated with these diseases in this part of the world. ${ }^{42,43}$ In the present study, there was a statistically significant improvement in the DLQI of both groups after use of cosmetic camouflage for all the parameters of symptoms and feelings, daily and leisure activities and personal relationships. These parameters represent important areas relating to QoL in a patient's routine and the improvement in scores directly demonstrates the benefits of cosmetic camouflage. Overall, the mean improvement in DLQI in the leprosy group with cosmetic camouflage $(14.67 \pm 3.87)$ was much higher than that observed in the vitiligo group $(8.64 \pm 2.96)$, showing how leprosy patients experienced greater benefit from this intervention (Figure 1).

\section{EASE OF USE AND EFFECTIVENESS}

All the leprosy patients in the study accepted cosmetic camouflage readily. The training and demonstration of its use in the clinic contributed to its acceptance. ${ }^{19}$ None of the patients in our study had any allergic/irritant reaction to cosmetic creams on application. The cost involved in the use of cosmetic camouflage is not very high. For example, the cost of cosmetic camouflage for covering a patch of size of a palm daily would be about INR 750 ( $\$ 10$ US dollars) a month. These products are readily available in India and the world over, for direct purchase from the market, as well as for order on internet.

The benefit of cosmetic camouflage was equally good in patients of both groups. A special mention must be made of two young patients, one from Chennai (Figure 5), India and the other a visitor from the US with patches of 'BT in type 1 lepra reaction' on the face (Figure 3). Both were working in the corporate sector and were very hesitant and ill at ease about attending the office because of the obvious erythematous facial patches. With camouflage they were extremely happy, as they could continue their vocation without social embarrassment while ensuring regular treatment as needed.

It is important to note that stigma in leprosy not only affects the individuals and their families but also impacts the effectiveness of public health programmes for leprosy. ${ }^{43,44}$ Stigma creates fear and patients fail to seek early treatment resulting in further spread of the disease and disabilities in the individual, which compounds stigma. ${ }^{45}$ It should be noted that fear and 
stigma are difficult to remove and can only be dealt with through a combination of strategies. ${ }^{46}$ Use of camouflage, to change the image of the disease by masking skin lesions is a simple, repeatable daily intervention to reduce the anxiety, improve DLQI and to successfully address stigma. As cosmetic camouflage is a widely accepted modality to improve the QoL in patients with vitiligo, the same may be recommended to patients with leprosy skin patches.

Limitations of the study were the small sample size, short observation period, noninclusion of lepromatous patients with diffuse infiltration and those with clofazimine induced pigmentation.

\section{Conclusions}

Cosmetic camouflage is a text book prescribed management option for vitiligo over decades for both children and adults to help them psychologically by improving life quality indices. However, for leprosy many papers document the effects of stigma, but few discuss solutions ${ }^{7}$ or strategies to improve appearance. In the present study, DLQI was shown to be improved significantly in both leprosy and vitiligo patients with the use of cosmetic camouflage. The study also observed that application of cosmetic camouflage is a simple, well-accepted, inexpensive daily use method to improve skin appearance in leprosy patients to reduce the stigma associated with visible skin lesions. Hence, the present study advocates the use of cosmetic camouflage for visible skin lesions in leprosy as an important strategy to improve QoL and to fight against stigma associated with this dreaded disease.

\section{Conflict of Interest}

The authors report no conflicts interest.

\section{Ethics approval}

Obtained from the institutional Ethics committee of Bhaskar medical college, Yenkapally, RR district, where this work was done.

\section{Data availability statement}

This study did not utilize data not already in the public domain.

\section{Funding information}

This study was fully funded by a Research grant from Indian Association of Dermatology Venereology and leprology (IADVL).

\section{Patient consent}

Fully informed written consent was obtained from all patients of this study, including for the use of clinical photographs in publication with all due precautions.

\section{Clinical Trials registration}

No trials registration was required for this study.

\section{References}

1 Boehncke WH, Ochsendorf F, Paeslack I, Kaufmann R, Zollner TM. Decorative cosmetics improve the quality of life in patients with disfiguring skin diseases. Eur J Dermatol, 2002; 12: 577-580. 
2 Das NK, De A, Naskar B, Sil A, Das S, Sarda A, Chatterjee G. A quality of life study of patients with Leprosy attending the dermatology OPD of a tertiary care center of Eastern India. Indian J Dermatol, 2020; 65: 42-46.

3 Govindharaj P, Srinivasan S, Darlong J. Quality of life of persons affected by leprosy in an endemic district, West Bengal, India. Indian J Dermatol, 2018; 63: 459-464.

4 Barrett R. Self-mortification and the stigma of leprosy in northern India. Med Anthropol Q, 2005; 19: $216-230$.

5 Postage's H. Leprosy, the key to another kingdom. Lepr Rev, 2011; 82: 155-167.

6 Chaturvedi SK, Singh G, Gupta N. Stigma experience in skin disorders: an Indian perspective. Dermatol Clin, 2005; 23: 635-642.

7 Rafferty J. Curing the stigma of leprosy. Lepr Rev, 2005; 76: 119-126.

8 Thompson A, Kent G. Adjusting to disfigurement: processes involved in dealing with being visibly different. Clin Psychol Rev, 2001; 21: 663-682.

9 Finlay AY, Khan GK. Dermatology Life Quality Index (DLQI) - a simple practical measure for routine clinical use. Clin Exp Dermatol, 1994; 19: 210-216.

10 Finlay AY. Quality of life indices. Indian J Dermatol Venereol Leprol, 2004; 70: 143-148.

11 Holm EA, Wulf HC, Stegmann H, Jemec GB. Life quality assessment among patients with atopic eczema. $\mathrm{Br} J$ Dermatol, 2006; 154(4): 719-725.

12 Dolatshahi M, Ghazi P, Feizy V, Hemami MR. Life quality assessment among patients with vitiligo: Comparison of married and single patients in Iran. Indian J Dermatol Venereol Leprol, 2008; 74: 700.

13 Parsad D, Pandhi R, Dogra S, Kanwar AJ, Kumar B. Dermatology life quality index score in vitiligo and its impact on the treatment outcome. Br J Dermatol, 2003; 148: 373-374.

14 Sangma LN, Nath J, Bhagabati D. Quality of life and psychological morbidity in vitiligo patients: A study in a teaching hospital from North-East India. Indian J Dermatol, 2015; 60: 142.

15 Ongenae K, Dierckxsens L, Brochez L, van Geel N, Naeyaert JM. Quality of life and stigmatization profile in a cohort of vitiligo patients and effect of the use of camouflage. Dermatology, 2005; 210: 279-285.

16 Holme SA, Beattie PE, Fleming CJ. Cosmetic camouflage advice improves quality of life. Br J Dermatol, 2002; 147: 946-949.

17 Kryolan professional makeup - dermacolor; c2020 [cited2020 June 18]. Available from https://in.kryolan.com /product-lines/dermacolor/palettes.

18 Rayner VL. Cosmetic rehabilitation. Dermatol Nurs, 2000; 12(4): 267-271.

19 Kaliyadan F, Kumar A. Camouflage for patients with vitiligo. Indian J Dermatol Venereol Leprol, 2012; 78: 8-15.

20 Antoniou C, Stefanaki C. Cosmetic camouflage. J Cosmet Dermatol, 2006; 5: 297-301.

21 Bouloc A. Chapter 21: Camouflage techniques. In: Draelos ZD (ed.), Cosmetic Dermatology: Products and Procedures. New Jersey: John Wiley \& Sons, 2015; pp. 186-192.

22 Hayashi N, Imori M, Yanagisawa M, Seto Y, Nagata O, Kawashima M. Make-up improves the quality of life of acne patients without aggravating acne eruptions during treatments. Eur J Dermatol, 2005; 15(4): $284-287$.

23 Dharmendra, Chatterjee SN. Maculo-anaesthetic leprosy its diagnosis and classification. Lepr Rev, 1962; 33: 106-118.

24 Rawalani SM, Gummadapu S, Motwani M, Bhowate R, Rawalani S. Orofacial lesions in treated central Indian leprosy patients: a cross sectional study with review of literature. Indian J Lepr, 2008; 80: 161-165.

25 Hogeweg M, Kiran KU, Suneetha S. The significance of facial patches and type I reaction for the development of facial nerve damage in leprosy. A retrospective study among 1226 paucibacillary leprosy patients. Lepr Rev, 1991; 62: 143-149.

26 Pallagatti S, Sheikh S, Kaur A, Aggarwal A, Singh R. Oral cavity and leprosy. Indian Dermatol Online J, 2012; 3(2): 101-104, doi:10.4103/2229-5178.96700; PMID: 23130281; PMCID: PMC3481884.

27 Kumar B, Dogra S, Kaur I. Epidemiological characteristics of leprosy reactions: 15 years experience from north India. Int J Lepr Other Mycobact Dis, 2004; 72(2): 125-133.

28 van Brakel WH. Measuring leprosy stigma - a preliminary review of the leprosy literature. Int J Lepr Other Mycobact Dis, 2003; 71: 190-197.

29 van Brakel W. Measuring leprosy stigma - a preliminary review of the leprosy literature. Int J Lepr Other Mycobact Dis, 2003; 71: 190-197.

30 WHO Global leprosy strategy 2016-2020: accelerating towards a leprosy-free world. Operational manual. Geneva. 2016.

31 Salsberg JM, Weinstein M, Shear N, Lee M, Pope E. Impact of cosmetic Camouflage on the quality of life of children with skin disease and their families. J Cutan Med Surg, 2016; 20: 211-215.

32 Heijnders M, van Der Meij S. The fight against stigma: an overview of the stigma reduction strategies and interventions. Psychol Health Med, 2006; 11: 353-363.

33 Antoniou C, Stefanaki C. Cosmetic camouflage. J Cosmet Dermatol, 2006; 5: 297-301. 
34 Singh SM, Narang T, Dogra S, Verma AK, Gupta S, Handa S. An analysis of dermatological quality-of-life scores in relation to psychiatric morbidity in psoriasis. Indian Dermatol Online J, 2016; 7: 208-209.

35 Charan UP, Peter CD, Pulimood SA. Impact of hand eczema severity on quality of life. Indian Dermatol Online $J, 2013 ; 4:$ 102-105.

36 Stevelink SA, van Brakel WH, Augustine V. Stigma and social participation in Southern India: differences and commonalities among persons affected by leprosy and persons living with HIV/AIDS. Psychol Health Med, 2011; 16(6): 695-707, doi:10.1080/13548506.2011.555945.

37 Luka EE. Understanding the stigma of leprosy. SSMJ Vol 3 Issue 3 August 2010. [cited 2020 June 18]. Available from: http://www.southsudanmedicaljournal.com/assets/files/Journals/vol_3_iss_3_aug_10/Leprosy\%20stigm a.pdf.

38 Sawant NS, Vanjara NA, Khopkar U. Gender Differences in Depression, Coping, Stigma, and Quality of Life in Patients of vitiligo. Dermatology research and Practice, Hindawi. 2019; ID 6879412, doi:10.1155/2019/6879412.

39 Krüger C, Schallreuter K. Stigmatisation, avoidance behaviour and difficulties in coping are common among adult patients with vitiligo. Acta Derm Venereol, 2015; 95(5): 553-558.

40 Pichaimuthu R, Ramaswamy P, Bikash K, Joseph R. A measurement of the stigma among vitiligo and psoriasis patients in India. Indian J Dermatol Venereol Lep, 2011; 77: 300-306.

41 Bae JM, Lee SC, Kim TH, Yeom SD, Shin JH, Lee WJ. Factors affecting the quality of life in patients with vitiligo: a nationwide study. Br J Dermatol, 2018; 178: 238-244.

42 Pahwa P, Mehta M, Khaitan BK, Sharma VK, Ramam M. The psychosocial impact of vitiligo in Indian patients. Indian J Dermatol Venereol Lep, 2013; 79: 679-685.

43 Rao PSS. Perspectives on the impact of stigma in leprosy: strategies to improve access to health care. Res Rep Trop Med, 2015; 6: 49-57.

44 Van Brakel WH. Measuring health-related stigma-a literature review. Psychol Health Med, 2006; 11(3): 307334.

45 Barrett R. Self-mortification and the stigma of leprosy in northern India. Med Anthropol Q, 2005; 19(2): 216230.

46 Kent G. Testing a model of disfigurement: effects of a skin camouflage service on well-being and appearance anxiety. Psychol Health Med, 2002; 17: 377-386. 